\title{
Sub-Picosecond Pulse Generation from a Laser-Diode-Pumped, Self-Starting Additive-Pulse Mode-Locked Nd:LMA Laser
}

\author{
D. W. Hughes, A. A. Majdabadi, J. R. M. Barr, and D. C. Hanna \\ Optoelectronics Research Centre, Southampton University, Southampton, \\ SO9 5NH, United Kingdom
}

\begin{abstract}
We report the generation of sub-picosecond modelocked pulses at $1.05 \mu \mathrm{m}$ from an additive-pulse modelocked laser-diode-pumped $\mathrm{La}_{1-x} \mathrm{Nd}_{x} \mathrm{MgAl}_{11} \mathrm{O}_{19}$ laser. The repetition rate was $93 \mathrm{MHz}$, and the maximum average output power was $60 \mathrm{~mW}$.
\end{abstract}

\section{$\underline{\text { Introduction }}$}

In recent years, the use of a nonlinear element in an external coupled cavity (additive-pulse mode-locking, APM, or coupled-cavity mode-locking) has become recognised as a well-established technique for the generation of ultrashort pulses from a variety of laser materials. These include colour centre lasers [1], Ti:sapphire lasers [2] and a number of neodymium doped systems including Nd:YAG [3,4], Nd:YLF [5], Nd:glass [6] and $\mathrm{La}_{1-x} \mathrm{Nd}_{\mathrm{MgAl}} \mathrm{Mg}_{11} \mathrm{O}_{19}$ [7] (Nd:LMA. or LNA). In the majority of these systems the use of the APM technique has resulted in the generation of the shortest pulses reported to date from the particular active medium.

Several APM systems have been operated using laser diodes as the pump source eg $[3,5]$. The use of laser diode pumping in conjunction with the APM technique allows the construction of highly reliable, robust, compact and efficient solid state sources of ultrashort pulses. We have previously reported the generation of 550 fsec pulses from an additive-pulse mode-locked Nd:LMA laser pumped by a Ti:sapphire laser [7]. In this paper we will present results obtained from a laserdiode-pumped Nd:LMA laser.

The Nd:LMA laser was first reported in the mid 1980 s, since when a number of groups have reported its performance [7-12]. It has been pumped using ion lasers (both argon ion and krypton ion operating at $514 \mathrm{~nm}$ and $752 \mathrm{~nm}$ respectively) [8], by a Ti:sapphire laser operating at $789 \mathrm{~nm}[7,9]$, and by laser diodes operating in the region of $800 \mathrm{~nm}[10,11]$. Its mode-locked operation has been reported using the saturable absorber mode-locking of a flashlamp pumped system [12] and the active (frequency modulation) mode-locking of a laser-diode-pumped system [11]. Pulse durations of 10 psec and 14 psec respectively were obtained.

Nd:LMA is a laser material which lends itself especially well to both pumping with laser diodes and ultrashort pulse generation via mode-locking techniques. It has a broad absorption band centred around $800 \mathrm{~nm}$, which means that it can be readily pumped by commercially available AlGaAs laser diodes. The fact that the absorption band is so broad also means that precise temperature control of the laser diode wavelength is not required. The fluorescence spectrum of Nd:LMA around $1 \mu \mathrm{m}$ shows two peaks, centred at $1.054 \mu \mathrm{m}$ and $1.082 \mu \mathrm{m}$. The broad linewidth of these peaks (1.3 $\mathrm{THz}$ and $2.2 \mathrm{THz}$ respectively) means that sub-picosecond mode-locked pulses can be supported at both of these wavelengths.

In our work, we are particularly interested in the $1.054 \mu \mathrm{m}$ emission wavelength. The laser is being developed to be used as a seed source for chirped pulse amplification (CPA) experiments using the VULCAN Nd:glass laser facility at the Rutherford Appleton Laboratory [13]. A clear prerequisite for such a source is that its wavelength must coincide with the peak of the gain curve in the amplifier chain, which is $1.054 \mu \mathrm{m}$. Furthermore, the source must be laser-diode-pumped, the individual pulse energy must exceed $0.5 \mathrm{~nJ}$, and the pulses must be of less than 1 psec duration. Clearly, in principle at least, the APM laser-diode-pumped 
Nd:LMA laser is capable of meeting these criteria. Currently an APM laser-ciiode-pumped Nd:YLF laser, which generates mode-locked pulses of around 1.5 to 2 psec is being used as the seed source.

One further candidate which must be considered as a potential seed laser is the APM laser-diode-pumped Nd:glass laser. This laser has previously been demonstrated to generate pulse durations in the region of 600 to $700 \mathrm{fsec}$ [14], and with its fluorescence linewidth of $5.3 \mathrm{THz}$ can clearly support considerably shorter pulses. However, we have previously found that Nd:glass is susceptible to a range of thermal problems (in particular thermally induced birefringence and melting) due to its low melting point $\left(\sim 550^{\circ} \mathrm{C}\right)$ and thermal conductivity $(\sim 0.5 \mathrm{~W} / \mathrm{m} / \mathrm{K})[14,15]$. These problems make the Nd:glass laser difficult to scale to high powers. We have observed no catastrophic thermal problems in the use of the laser-diode-pumped Nd:LMA laser.

\section{Experimental Work}

The experimental apparatus is shown in figure 1.

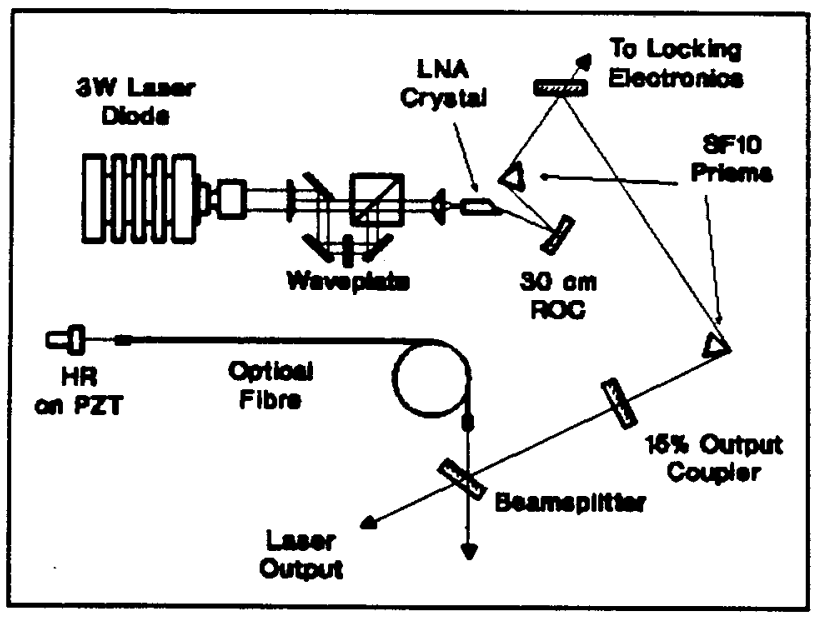

Figure 1. Schematic diagram of the experimental apparatus

The pump source was a $3 \mathrm{~W}$ laser diode array (SDL 2482). Its wavelength was temperature controlled using a thermoelectric cooler to ensure good absorption of the pump radiation in the active medium. The pump beam was collimated in the plane perpendicular to the junction (the vertical plane) by a compound lens of focal length $6.5 \mathrm{~mm}$. All the optics used in the collimation and focusing apparatus were anti-reflection (AR) coated at the pump laser wavelength. A $15 \mathrm{~cm}$ cylindrical lens was used to collimate the pump beam in the plane of the junction (the horizontal plane).

After collimation, the pump beam was split into two. One arm was then passed through a waveplate before being overlapped with the other arm at a polarizing beamsplitting cube. The recombined beam was then focused into the active medium using a $3.2 \mathrm{~cm}$ spherical lens. The reason for this splitting and recombination was to try to improve the quality of the pump beam, in particular its divergence, near the focus of the $3.2 \mathrm{~cm}$ lens (in the plane of the laser diode which is nondiffraction limited, the horizontal plane). It was found that, without splitting the beam, a minimum spot size (diameter) in the horizontal plane of approximately 200 $\mu \mathrm{m}$ could be achieved. With the beam split, it was found that each half of the beam independently could be focused to slightly less than $200 \mu \mathrm{m}$. The divergence of the beam was thus reduced by a factor of around 2 . Figure 2 shows the variation of one half of the split beam in the focal plane of the $3.2 \mathrm{~cm}$ lens. It can be seen that, as is to be expected, the focused beam was considerably larger in the horizontal plane than in the vertical plane. The average spot size (diameter) of the laser cavity TEM $_{\infty}$ mode was calculated to be $204 \mu \mathrm{m}$ in the horizontal plane and $116 \mu \mathrm{m}$ in the vertical plane.

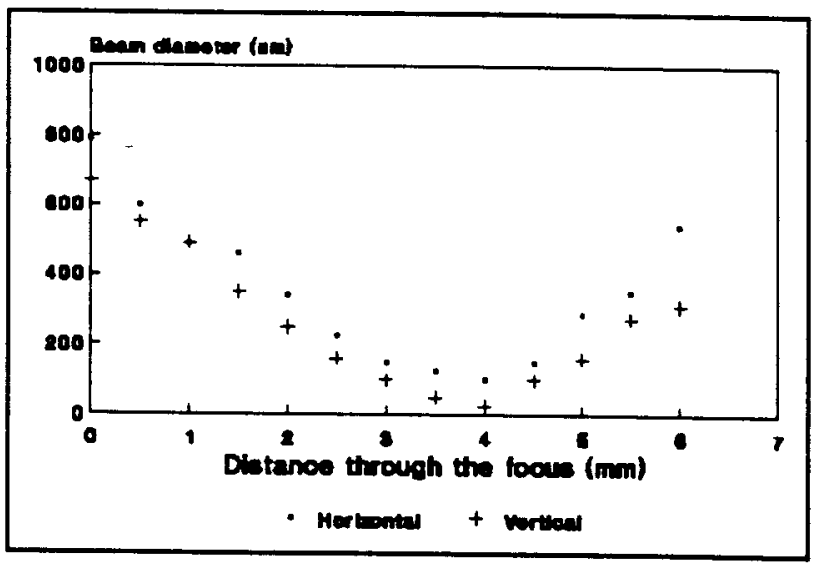

Figure 2. Variation of the pump beam spot size in the focal plane of the focusing lens.

The active medium was a $9 \mathrm{~mm}$ long, $5 \mathrm{~mm}$ diameter plane-Brewster rod of Nd:LMA with a $9 \%$ wt. doping of $\mathrm{Nd}$ ions. The plane surface of the rod was coated to give high transmission at the pump laser wavelength, and high reflectivity at the laser wavelength. The cavity was designed to compensate for the astigmatism caused by the Brewster angle of the laser rod, and was completed using a $30 \mathrm{~cm}$ radius of curvature (ROC) turning mirror and either a $90 \%$ or $85 \%$ reflectivity output coupler. In addition, a pair of Brewster angled 
SF10 prisms separated by $92 \mathrm{~cm}$ were inserted in the cavity to compensate for the net positive dispersion of the coupled cavity scheme. The total cavity length was $1.4 \mathrm{~m}$. With this arrangement, without the prisms in the cavity and with a $10 \%$ output coupler, the laser performance is shown in figure 3 . It can be seen that the laser performance (in particular the slope efficiency) was significantly improved when the splitting scheme was adopted. On insertion of the prisms in the cavity, the laser output power was reduced by around $12 \%$. The laser output was at all times single transverse mode.

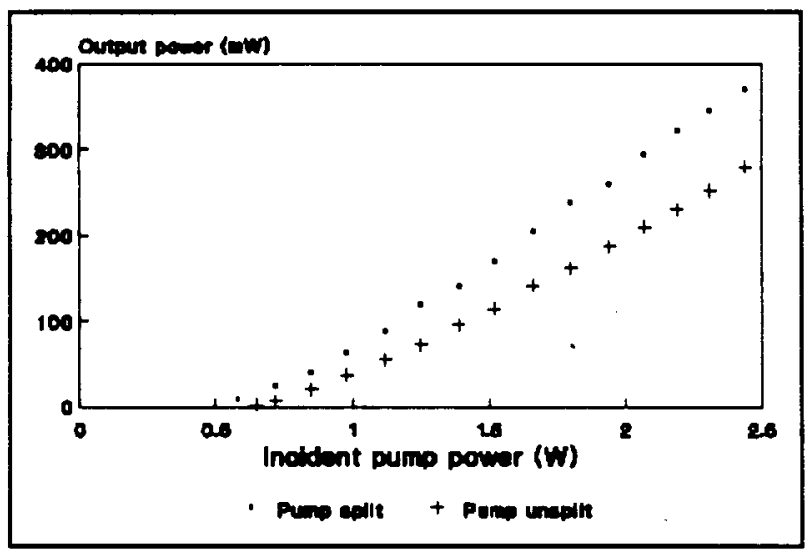

Figure 3. Comparison of the laser performance with the pump beam split and unsplit

The external coupled cavity was formed using a 95\% reflectivity beam splitter to direct a portion of the laser output into a single mode optical fibre of length $1.55 \mathrm{~m}$. GRIN lenses were used to couple into and out of the optical fibre, and coupling efficiencies in the region of 65 to $70 \%$ were readily obtained. Index matching at the fibre to GRIN lens interfaces was accomplished using a drop of paraffin. The coupled cavity was then completed using a high reflector HR mounted on a piezoelectric stack PZT. The total length of the coupled cavity was twice that of the main laser cavity. These initial parameters (long fibre length, high beamsplitter reflectivity) were chosen to facilitate self-starting APM operation, and, as will be seen later, were not optimized.

With the cavity lengths correctly matched, modelocking was readily achieved. The relative phases of the two cavities were locked at the correct value for modelocking using the technique of Mitschke and Mollenauer [16], and stable mode-locking was observed at the laser round trip frequency of $107 \mathrm{MHz}$. Mode-locking was observed for laser powers as low as $36 \mathrm{~mW}$ launched into the fibre. The pulse duration was measured using a standard interferometric autocorrelation technique. A typical autocorrelation trace is shown in figure 4. The pulse duration corresponding to this trace was 750 fsec assuming a hyperbolic secant temporal profile. The optical spectrum corresponding to this autocorrelation is shown in figure 5, and has a bandwidth (FWHM) of $430 \mathrm{GHz}$. The time-bandwidth product is thus 0.32 , very close to the transform limited value for a hyperbolic secant pulse $(0.315)$. The maximum average output power from the laser was $18 \mathrm{~mW}$, which corresponds to a pulse energy of $0.17 \mathrm{~nJ}$. Without the prisms in the cavity, strongly chirped puises of 1 to 2 psec duration were obtained, indicating the necessity for intracavity dispersion compensation.

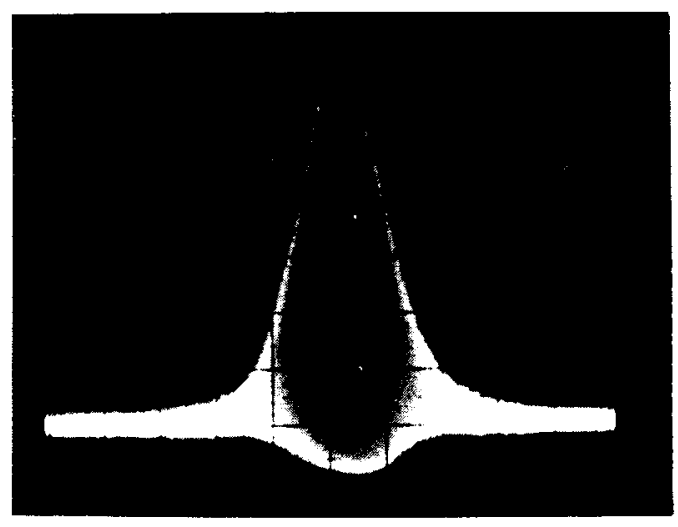

Figure 4. Interferometric autocorrelation trace. Time Delay $660 \mathrm{fsec} / \mathrm{div}$

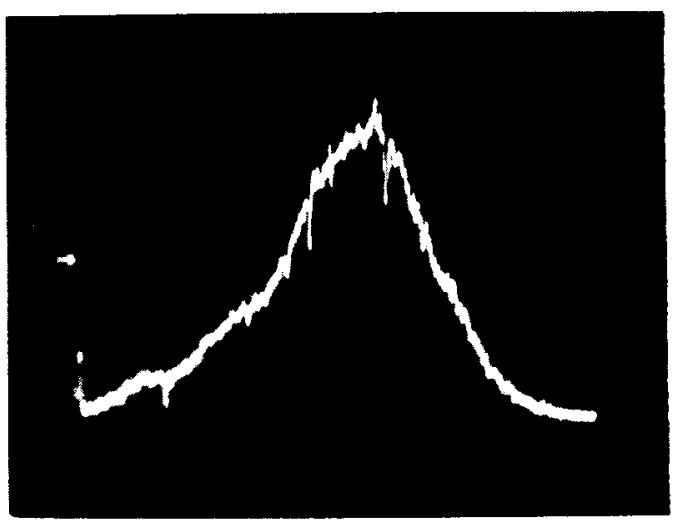

Figure 5. Optical spectrum of the mode-locked laser corresponding to the autocorrelation trace shown in figure 4. Horizontal Scale: $150 \mathrm{GHz} / \mathrm{div}$

In order to try to optimize the system, several changes were made to the apparatus. Firstly, the length of the fibre was reduced to $82 \mathrm{~cm}$, which meant that the ratio of the main cavity length to the external cavity length was $1: 1$. Secondly, the output coupler reflectivity was reduced to $85 \%$. Furthermore, a range of 
beamsplitter reflectivities varying from $85 \%$ to $95 \%$ were used. With the $85 \%$ output coupler, a maximum average output power of $290 \mathrm{~mW}$ could be obtained before the output beam was split.

With this experimental set-up, significantly shorter pulses were obtained. A typical autocorrelation trace obtained when the $95 \%$ beamsplitter was used is shown in figure 6. Again assuming a hyperbolic secant temporal profile, the pulse duration is $420 \mathrm{fsec}$. The corresponding optical spectrum is shown in figure 7, and has a bandwidth of $750 \mathrm{GHz}$ (FWHM). The timebandwidth product is thus 0.32 , indicating near transform limited pulses.

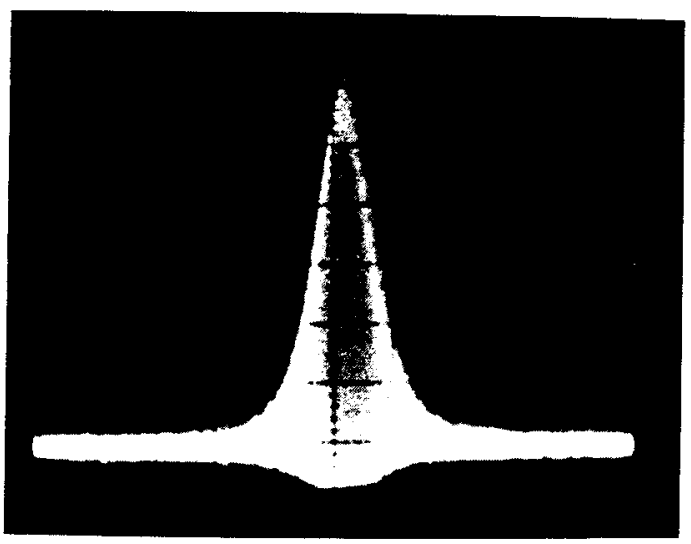

Figure 6. Interferometric autocorrelation trace. Time Delay: 600 fsec/div

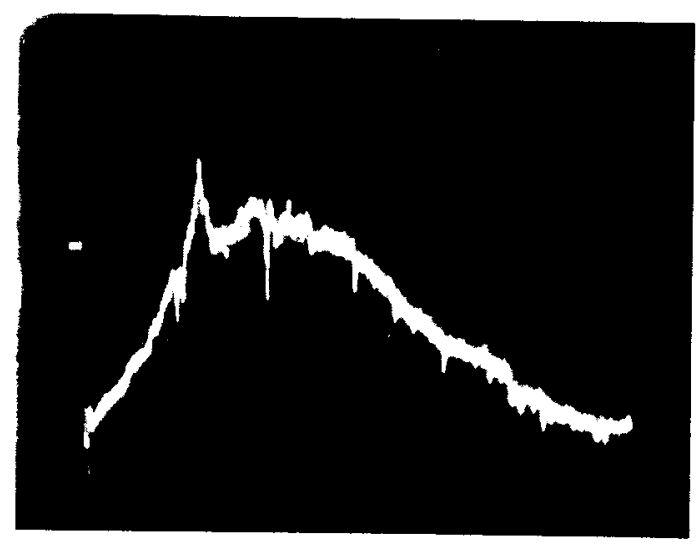

Figure 7. Optical spectrum corresponding to the autocorrelation trace shown in figure 6. Horizontal Scale: $150 \mathrm{GHz} / \mathrm{div}$

When the $95 \%$ reflectivity beamsplitter was used, the maximum useful average output power was $18 \mathrm{~mW}$, corresponding to a pulse energy of $0.19 \mathrm{~nJ}$. The threshold for self-starting operation was $30 \mathrm{~mW}$ coupled into the fibre. Use of the lower reflectivity beamsplitters resulted in slightly longer pulse durations (500 to 600 fsec) and average output powers as high as $60 \mathrm{~mW}$. This corresponds to a pulse energy as high as $0.65 \mathrm{~nJ}$.

\section{Conclusions and Future Work}

In conclusion, we have demonstrated a laser-diodepumped additive-pulse mode-locked Nd:LMA laser capable of generating pulses as short as $420 \mathrm{fsec}$, and sub-picosecond pulses of energies as high as $0.65 \mathrm{~nJ}$. These shortest pulses which were obtained are only a little longer than the bandwidth limited value of $350 \mathrm{fsec}$ for Nd:LMA, so that further significant decreases in pulse duration are unlikely. Future work will thus concentrate on obtaining higher output powers from the system. In the first place, this could be achieved by simply reducing the beamsplitter reflectivity. Significant power increases may be obtained by pumping with two $3 \mathrm{~W}$ laser diode arrays. Initial experiments have shown that while Nd:LMA exhibits no catastrophic thermal problems ie melting or other material damage, thermal problems (in particular thermal lensing) do exist at these high pump powers. It may well be that more careful consideration of cavity design, better heat sinking of the laser rod and use of a lower dopant sample could help to yield stable high power operation of this material. If this proves possible to achieve, then the high intracavity powers which would be obtained suggest that the next logical step would be the development of a Kerr lens mode-locked (KLM) [17] laser-diode-pumped Nd:LMA laser. This technique has already proved successful in mode-locking a laser-diode-pumped Nd:YLF laser [18]. KLM systems have several advantages over APM systems, in particular the reduced complexity compared to the coupled cavity arrangement, and the fact that there is no need to tap off any of the laser output power into an external coupled cavity.

\section{Acknowledoments}

The authors are grateful to Dr Ch Wyon of LETI, Grenoble, France, for supplying us with the Nd:LMA rod used in this experiment. This work was supported by a Science and Engineering Research Council funded collaboration with the Rutherford Appleton Laboratory.

\section{References}

1. J. Mark, L. Y. Liu, K. L. Hall, H. A. Haus and E. P. Ippen, "Femtosecond pulse generation in a laser with a nonlinear external resonator" Opt. Lett.14, 48-50 (1989)

2. J. Goodberlet, J. Wang, J. G. Fujimoto and P. A. Schultz, "Femtosecond passively modelocked $\mathrm{Ti}: \mathrm{Al}_{2} \mathrm{O}_{3}$ laser with a nonlinear external cavity" Opt. Lett.14, 1125-1127 (1989) 
3. J. Goodberlet, J.-Jacobson, J. G. Fujimoto, P. A. Schultz and T. Y. Fan, "Self-starting additive-pulse mode-locked diode-pumped Nd:YAG laser" Opt. Lett. 15, 504-506 (1990)

4. J. R. M. Barr and D. W. Hughes, "Coupled cavity modelocking of a Nd:YAG laser using second-harmonic generation" Appl. Phys. B49, 323-325 (1989)

5. G. P. A. Malcolm, P. F. Curley and A. I. Ferguson, "Additive-pulse mode locking of a diode-pumped Nd:YLF laser" Opt. Lett.15, 1303-1305 (1990)

6. F. Krausz, C. Spielmann, T. Brabec, E. Wintner and A. J. Schmidt, "Self-starting additive-pulse mode locking of a $\mathrm{Nd}$ :glass laser" Opt. Lett.15, 1082-1084 (1990)

7. M. W. Phillips, J. R. M. Barr, D. W. Hughes, D. C. Hanna, Z. Chang, C. N. Danson and C. B. Edwards, "Self-starting additive pulse mode-locking of a Nd:LMA laser" Opt. Lett.17, 1453-1455 (1992)

8. L. D. Schearer, M. Leduc, D. Vivien, A.-M. Lejus and J. Thery, "LNA: a new cw Nd laser tunable around 1.05 and 1.08. $\mu \mathrm{m}$ " IEEE J. Quantum Electron.OE-22, 713-717 (1986)

9. T. Y. Fan and M. R. Kokta, "End-pumped $\mathrm{Nd}: \mathrm{LaF}_{3}$ and $\mathrm{Nd}: \mathrm{LaMgAl}_{11} \mathrm{O}_{19}$ lasers" IEEE J. Quantum Electron.OE-25, 1845-1849 (1989)

10. J. Hamel, A. Cassimi, H. Abu-Safia, M. Leduc and L. D. Schearer, "Diode pumping of LNA lasers for helium optical pumping" Opt. Commun.63, 114-117 (1987)

11. D. W. Hughes, A. A. Majdabadi, J. R. M. Barr and D. C. Hanna, "An FM mode-locked, laser-diode-pumped $\mathrm{La}_{1 \cdot \mathrm{x}} \mathrm{Nd}_{x} \mathrm{MgAl}_{11} \mathrm{O}_{19}$ laser" submitted to Applied Optics (1992)
12. M. I. Demchouk, V. P. Mikhailov, A. K. Gilev, A. M. Zabaznov and A. P. Shkadarevich, "Investigation of the passive mode locking in a La-Nd-Mg hexaaluminatedoped laser" Opt. Commun.55, 33-34 (1985)

13. Z. Chang, C. N. Danson, M. D. Dooley, C. B. Edwards, M. H. R. Hutchinson, I. P. Mercer, M. R. G. Miller, Z. Najmudin, C. E. Ollman, D. A. Pepler, R. A. Smith, P. F. Taday, W. T. Toner and T. R. Winstone, "Ultra-short pulse operation of the VULCAN Nd:glass laser facility using chirped pulse amplification" Central Laser Facility Annual Report, Rutherford Appleton Laboratory (1992)

14. D. W. Hughes, M. W. Phillips, J. R. M. Barr and D. C. Hanna, "A laser-diode-pumped Nd:glass laser: mode-locked, high power and single frequency performance" IEEE J. Quantum Electron. OE-28, 1010-1017 (1992)

15. D. W. Hughes, J. R. M. Barr and D. C. Hanna, "A high power, high efficiency, laserdiode-pumped, continuous wave miniature Nd:glass laser" Opt. Commun. 84, 401-408 (1991)

16. F. M. Mitschke and L. F. Mollenauer, "Stabilizing the soliton laser" IEEE J. Quantum Electron.OE-22, 2242-2250 (1986)

17. D. K. Negus, L. Spinelli, N. Goldblatt and G. Feugnet, "Sub 100 fsec pulse generation by Kerr lens mode locking in $\mathrm{Ti}: \mathrm{Al}_{2} \mathrm{O}_{3}{ }^{\prime \prime}$ in Technical Digest of Advanced Solid State Lasers (Optical Society of America, Washington, DC, 1991), paper PDP4-1

18. G. P. A. Malcolm and A. I. Ferguson, "Selfmode locking of a diode-pumped Nd:YLF laser" Opt. Lett.16, 1967-1969 (1991) 